\title{
Polypills with or without aspirin for primary prevention of cardiovascular disease
}

Ischaemic heart disease and stroke are the conditions that contribute most to the global burden of disease, as measured by disability-adjusted life-years in people aged 50 years and older, ${ }^{1}$ despite many cardiovascular diseases being preventable. ${ }^{2,3}$ Two of the key modifiable risk factors are hypertension and high serum lipid concentrations. $^{2,3}$ There is great interest in simple approaches with wide applicability to address these risk factors, including using fixed-dose combination drug regimens, also known as polypills. This strategy was proposed in the early 2000s and was predicted by one proponent to reduce cardiovascular disease by more than 80\%. ${ }^{4}$ Although this original claim now seems overstated, evidence is accumulating from randomised controlled trials that polypills can reduce cardiovascular disease, but questions remain with regards to the balance of benefit and harm, the appropriate target population, and which drugs should be included in a polypill.

In The Lancet, Philip Joseph and colleagues ${ }^{5}$ report their individual participant data meta-analysis of three large outcome trials of fixed-dose combination strategies in the primary prevention of cardiovascular disease. More than 18000 participants (49.8\% women) were included in total, from 26 countries, with a median follow-up of 5 years. Joseph and colleagues specifically aimed to determine whether aspirin should be included in fixed-dose combinations, the size of effect on specific cardiovascular disease events, safety, and effects in different subgroups.

With regards to aspirin, the analysis provides indirect evidence that a fixed-dose combination including aspirin is more effective at preventing cardiovascular events than one without. Compared with the control group, the group receiving fixed-dose combination strategies including aspirin had a $47 \%$ reduction (hazard ratio [HR] 0.53, 95\% $\mathrm{Cl} 0.41-0.67, \mathrm{p}<0.0001$; number needed to treat [NNT] 37) in the primary outcome (a composite of cardiovascular death, myocardial infarction, stroke, or arterial revascularisation), compared with a $32 \%$ reduction $(0.68,0.57-0.81$, $\mathrm{p}<0.0001)$ for fixed-dose combination strategies without aspirin (NNT 66). This finding is consistent with the only direct randomised evidence from the TIPS-3 trial, which reported an HR of 0.79 (95\% Cl 0.63-1.00) for polypill versus placebo and of 0.69 (0.50-0.97) for polypill plus aspirin versus double placebo, ${ }^{6}$ and with the long established evidence base for the effectiveness of aspirin in prevention of cardiovascular disease. However, whether aspirin should be included in a fixeddose combination also requires consideration of other potential effects, both harmful and beneficial. Bleeding seems likely to have been underestimated number needed to harm [NNH] for fixed-dose combination with aspirin, 554), with only adverse events requiring hospitalisation likely to have been identified. In an earlier meta-analysis of the use of aspirin for primary prevention, which included negative trials of aspirin, the number needed to cause one major bleeding event was similar to that needed to prevent one cardiovascular event (NNH 210 vs NNT 241). ${ }^{8}$ However, the risks of aspirin could conceivably be mitigated in a polypill, because blood pressure lowering is associated with reduced risk of haemorrhagic stroke. ${ }^{9}$ Furthermore, aspirin is also associated with reduced risk of a number of cancer types. ${ }^{10}$

Overall, the observed effect of a fixed-dose combination strategy is substantial, with a $38 \%$ reduction ( $\mathrm{HR} 0.62,95 \% \mathrm{Cl} 0.53-0.73, \mathrm{p}<0.0001)$ in the risk of a primary cardiovascular disease outcome (NNT of 52 over 5 years), without significant adverse effects (with the exception of dizziness). The effects were similar in all subgroups, including people without hypertension or with normal lipid fractions, with a suggestion of a greater effect in older people, which would be further magnified if the higher absolute risk of events in this age group were taken into account. There is a striking consistency in the results of the three individual trials, despite the different drugs used, providing further confidence in the conclusion that a fixed-dose combination strategy is effective.

A fixed-dose combination strategy is an attractive option to reduce cardiovascular disease, along with non-pharmacological approaches. It avoids decisions on the use of blood pressure or lipid lowering therapies on the basis of the level of these risk factors. Many people with indications for pharmacotherapy, even those with
Published Online August 29, 2021 https://doi.org/10.1016/ PII See Online/Articles https://doi.org/10.1016/ S0140-6736(21)01827-4 
existing cardiovascular disease, in low-income and middle-income countries are not taking them. ${ }^{11}$ This suggests that strategies to initiate and maintain these drugs need to be as simple as possible. Such strategies might also have a role in high-income countries where the main alternative strategy (titrate treatment against risk factor levels) can result in undertreatment in practice. ${ }^{11}$ Cost-effectiveness analysis suggests that a fixed-dose combination strategy is potentially costeffective compared with treatment titration in a highincome setting. ${ }^{12}$

Although a polypill strategy might sit uncomfortably with precision medicine, there is now a substantial evidence base that such an approach is effective at reducing cardiovascular disease. Guideline writers and policy makers should consider how to incorporate this evidence base into guidelines and policies.

JM declares fees for academic advisory board membership for the Bristol-Myers Squibb-Pfizer-funded GUARD-AF trial of screening for atrial fibrillation, for Pfizersponsored education sessions on detection and diagnosis of atrial fibrillation and for advice on a heart failure app for Omron. RJM declares working with Omron on the development and evaluation of a blood pressure telemonitoring system for which consultancy and licencing fees are paid to his institution.

\section{* Jonathan Mant, Richard McManus jm677@medschl.cam.ac.uk}

Primary Care Unit, Department of Public Health \& Primary Care, University of Cambridge, Cambridge, UK (JM); Nuffield Department of Primary Care Health Sciences, University of Oxford, Oxford, UK (RM)

1 GBD 2019 Diseases and Injuries Collaborators. Global burden of
369 diseases and injuries in 204 countries and territories, 1990-2019: a systematic analysis for the Global Burden of Disease Study 2019. Lancet 2020; 396: 1204-22

2 O'Donnell MJ, Chin SL, Rangarajan S, et al. Global and regional effects of potentially modifiable risk factors associated with acute stroke in 32 countries (INTERSTROKE): a case-control study. Lancet 2016; 388: 761-75.

3 Yusuf S, Hawken S, Ounpuu S, et al. Effect of potentially modifiable risk factors associated with myocardial infarction in 52 countries (the INTERHEART study): case-control study. Lancet 2004; 364: 937-52.

4 Wald NJ, Law MR. A strategy to reduce cardiovascular disease by more than $80 \%$. BMJ 2003; 326: 1419

5 Joseph P, Roshandel G, Gao P, et al. Fixed-dose combination therapies with and without aspirin for primary prevention of cardiovascular disease: an individual participant data meta-analysis. Lancet 2021; published online Aug 29. https://doi.org/10.1016/S0140-6736(21)01827-4.

6 Yusuf S, Joseph P, Dans A, et al. Polypill with or without aspirin in persons without cardiovascular disease. N Engl J Med 2021; 384: 216-28

7 Antithrombotic Trialists' Collaboration. Collaborative meta-analysis of randomised trials of antiplatelet therapy for prevention of death, myocardial infarction, and stroke in high risk patients. BMJ 2002; 324: 71-86.

8 Zheng SL, Roddick AJ. Association of aspirin use for primary prevention with cardiovascular events and bleeding events: a systematic review and meta-analysis. JAMA 2019; 321: 277-87.

9 Woo D, Haverbusch M, Sekar P, et al. Effect of untreated hypertension on hemorrhagic stroke. Stroke 2004; 35: 1703-08

10 Qiao Y, Yang T, Gan Y, et al. Associations between aspirin use and the risk of cancers: a meta-analysis of observational studies. BMC Cancer 2018; 18: 288.

11 Yusuf S, Islam S, Chow CK et al. Use of secondary prevention drugs fo cardiovascular disease in the community in high-income, middle-income, and low-income countries (the PURE Study): a prospective epidemiological survey. Lancet 2011; 378: 1231-43.

12 Jowett $\mathrm{S}$, Barton $\mathrm{P}$, Roalfe $\mathrm{A}$ et al. Cost-effectiveness analysis of use of a polypill versus usual care of best practice for primary prevention in people at high risk of cardiovascular disease. PLoS One 2017; 12: e0182625. 